\title{
ENFERMAGEM E TECNOLOGIA: ANÁLISE DOS GRUPOS DE PESQUISA CADASTRADOS NA PLATAFORMA LATTES DO CNPq
}

\author{
Sayonara de Fátima Faria Barbosa', Grace Teresinha Marcon Dal Sasso², Isabel Berns ${ }^{3}$
}

\footnotetext{
${ }^{1}$ Doutora em Ciências da Saúde. Professor Adjunto do Departamento de Enfermagem da Universidade Federal de Santa Catarina (UFSC). Santa Catarina, Brasil. E-mail: sayonara@ccs.ufsc.br

${ }^{2}$ Doutora em Enfermagem. Professor Adjunto do Departamento de Enfermagem e do Programa de Pós-Graduação em Enfermagem da UFSC. Santa Catarina, Brasil. E-mail: grace@ccs.ufsc.br

${ }^{3}$ Aluna do Curso de Graduação em Enfermagem da USFC. Santa Catarina, Brasil. E-mail: bebeluberns@yahoo.com.br
}

RESUMO: Estudo descritivo que tem como objetivo analisar os grupos de pesquisas da área de Enfermagem, cadastrados na plataforma Lattes do site do Conselho Nacional de Desenvolvimento Científico e Tecnológico que apresentam dentre suas linhas de pesquisa, a produção de conhecimento sobre tecnologia. Por meio de levantamento de dados de todos os grupos de pesquisa em Enfermagem no Brasil cadastrados no Diretório de Grupos de Pesquisa do Conselho Nacional de Desenvolvimento Científico e Tecnológico, foram identificados 66 grupos, distribuídos por 29 instituições em todas as regiões do país. Mediante estatística descritiva foi observada uma maior concentração de grupos em instituições na região Sudeste do país. A maioria dos grupos aborda a tecnologia em suas várias formas, com destaque para sete grupos de pesquisa que contemplam a linha da Tecnologia da Informação e Comunicação como foco de sua produção.

DESCRITORES: Tecnologia. Pesquisa em enfermagem. Tecnologia da informação. Enfermagem.

\section{NURSING AND TECHNOLOGY: ANALYSIS OF THE RESEARCH GROUPS REGISTERED IN THE CNPq LATTES PLATFORM}

\begin{abstract}
The objective of this descriptive study was to analyze the nursing research groups registered in the Lattes platform of the site of Brazilian National Council for Scientific and Technological Development (CNPq) which present technology knowledge production amongst their lines of research. Through gathering the data available from all the nursing research groups in Brazil, recorded in the Directory of Research Groups of CNPq, 66 groups were identified, distributed throughout 29 institutions in all regions of the country. Descriptive statistics showed the greatest concentration of groups in institutions in the Southeastern region of the country. The majority of the groups approach technology in its various forms, with special focus given to seven research groups that contain the Information and Communication Technology as their main line of research production.
\end{abstract}

DESCRIPTORS: Technology. Nursing research. Information technology. Nursing

\section{ENFERMERÍA Y TECNOLOGÍA: ANÁLISIS DE LOS GRUPOS DE INVESTIGACIÓN REGISTRADOS EN LA PLATAFORMA LATTES DEL CNPq}

RESUMEN: Estudio descriptivo cuyo objetivo es analizar los grupos de investigación del área de enfermería que están registrados en la plataforma Lattes de la página web del Consejo Nacional para el Desarrollo Científico y Tecnológico, y que tienen entre sus líneas de investigación la producción del conocimiento en tecnología. A través de los datos obtenidos por medio de encuesta a todos los grupos de investigación de enfermería en el Brasil, que están registrados en el Directorio de los Grupos de Investigación del Consejo Nacional para el Desarrollo Científico y Tecnológico, se identificaron 66 grupos, distribuidos en 29 instituciones de todo el país. El análisis estadístico descriptivo permitió observar una mayor concentración de grupos en las instituciones de la región sudeste del país. La mayoría de los grupos se acerca a la tecnología en sus diversas formas, destacándose siete grupos de investigación que incluyen la línea de Tecnologías de la Información y Comunicación como centro de su producción.

DESCRIPTORES: Tecnología. Investigación en enfermería. Tecnología de la información. Enfermería. 


\section{INTRODUÇÃO}

A partir da década de 40, experenciou-se uma rápida expansão de conhecimentos e habilidades e, na forma de tecnologias se expressaram nas mais variadas áreas de atuação em saúde, dentre elas a pesquisa. ${ }^{1}$ Desta forma, na era da informação, o conhecimento é fator de sucesso e a medida do valor de qualquer disciplina e/ou organização. ${ }^{2}$

No Brasil, as atividades de produção de conhecimentos em pesquisa vêm sendo desenvolvidas por equipes de pesquisadores titulados ou em formação, organizados sob a designação de Grupos de Pesquisa.

$\mathrm{Na}$ Enfermagem brasileira estes grupos surgiram na década de 1970, e seu crescimento e forma de organização vêm se expandindo de modo significativo. ${ }^{3}$ Existem aproximadamente 323 grupos cadastrados no Diretório de Pesquisa do Conselho Nacional de Desenvolvimento Científico e Tecnológico (CNPq).

É importante ressaltar, que além de valorizar a produção do conhecimento, o Diretório dos Grupos de Pesquisa do CNPq passou a ser referência como medida desta produção de conhecimento, aqui se destacando a Enfermagem. Ou seja, a pesquisa em enfermagem se configura em um termo mensurável da produção do seu conhecimento no Brasil.

Apesar do grande foco humanista da Enfermagem, o avanço tecnológico em suas múltiplas expressões vem ganhando espaço na área de conhecimento da Enfermagem, o que leva ao interesse pelos estudos e pela produção de conhecimentos sobre o tema.

Entender a concepção de tecnologia em enfermagem é um desafio para uma profissão cujo foco histórico é o ser humano. Entretanto, a tecnologia não pode ser entendida como um paradigma de cuidado oposto ao humano, mas, sobretudo, um agente e objeto em função da pessoa humana. A tecnologia pode ser um fator que humaniza, mesmo nas arenas mais tecnologicamente intensas de cuidado em saúde. As dualidades da tecnologia como produto e significado, matéria e significado, produto e processo, repousam não em sua oposição necessária à humanização, mas, especialmente, em suas recursividades, isto é, na sua existência como ambas, força material objetiva e como uma realidade dinâmica e construída socialmente. ${ }^{1}$

De modo a compreender a produção científica da Enfermagem com foco em tecnologia, no Brasil, este estudo tem como objetivo analisar os grupos de enfermagem cadastrados na Plataforma
Lattes do $\mathrm{CNPq}$ que possuem linha de pesquisa com foco em tecnologia. Dessa temática geral emergiram as seguintes perguntas: quantos são os grupos de pesquisa de enfermagem que englobam a tecnologia entre suas linhas de pesquisa, no Brasil? Quem e quantos são os estudiosos do tema na área da Enfermagem? Em que estados se localizam e em quais regiões estão mais concentrados estes grupos? Qual a abordagem da tecnologia nos grupos de pesquisa?

Para a realização deste estudo foi utilizado o Diretório dos Grupos de Pesquisa, ${ }_{1}^{4}$ que é um projeto desenvolvido pelo CNPq desde 1992 e, mantém essencialmente as mesmas definições básicas e objetivos desde então. Suas bases contêm informações capazes de descrever os limites e o perfil geral da atividade científico-tecnológica no Brasil. São capazes de fornecer aos interessados um grande volume de informações diversificadas sobre detalhes de quem realiza as atividades, como e onde as realiza e, sobre o quê. Especificamente em relação a esta pesquisa, foi possível compreender elementos sobre o estágio atual em que se encontra a produção de conhecimento sobre tecnologia em enfermagem conforme pode ser observado na apresentação e discussão dos resultados.

\section{MÉTODOS}

A pesquisa descritiva foi realizada por meio do levantamento dos dados de todos os grupos de pesquisa em enfermagem do Brasil cadastrados no Diretório dos Grupos de Pesquisa CNPq, utilizando-se a palavra chave tecnologia ou tecnologias na busca em sua base corrente. Os passos para a coleta documental desta pesquisa descritiva foram os seguintes:

- acesso ao site institucional do CNPq (http://www.cnpq.br);

- em seguida, acesso ao tópico Diretório dos Grupos de Pesquisa, no qual consta o item Consulta e, posteriormente, o item Base Corrente;

- na tela da Base Corrente foi selecionada a opção Grupos e inseridas na caixa de pesquisa as palavras-chave tecnologia ou tecnologias;

- no filtro de busca, foi selecionada apenas a área do grupo Enfermagem.

Este procedimento possibilitou a identificação dos grupos de pesquisa em enfermagem do Brasil, cadastrados no site que se relacionam com a temática tecnologia, a partir de suas respectivas linhas de pesquisa, o que permitiu o acesso às informações de cada grupo. 
Em uma primeira consulta, foram identificados 73 grupos. Destes, foram excluídos sete por não apresentarem qual o objetivo da linha de pesquisa que estaria relacionada com o estudo da temática tecnologia, sendo então selecionados 66 grupos para análise. Todos os dados de cada um dos grupos de pesquisa foram registrados e analisados por estatística descritiva mediante cálculos de frequência absoluta e relativa.

Alguns dados como o número de mestres e doutores, foram obtidos por meio do acesso ao Currículo Lattes dos pesquisadores. Este acesso foi possível ao selecionar o nome do pesquisador na própria página do grupo, onde um link conduziu para o respectivo currículo.

Em alguns casos, embora a palavra tecnologia ou tecnologias fosse citada como parte do nome do grupo e/ou na linha de pesquisa, não estava explícito no objetivo do grupo o tipo da abordagem. Em outros casos, mesmo sem que o termo tecnologia ou tecnologias fizesse parte do grupo e/ ou na linha de pesquisa, foi possível estabelecer alguma relação com este tema.

\section{RESULTADOS E DISCUSSÕES}

Com os dados coletados no Diretório dos Grupos de Pesquisa do site do CNPq, foram identificados 66 grupos de pesquisa, distribuídos em 29 instituições de ensino superior públicas e privadas em enfermagem que se distribuem nas regiões brasileiras (Tabela 1 ).

Tabela 1 - Grupos de pesquisa em enfermagem com linha de pesquisa em tecnologia, por região do Brasil em 2008

\begin{tabular}{lcc}
\hline Região & $\mathbf{n}$ & $\mathbf{\%}$ \\
\hline Norte & 2 & 3 \\
Nordeste & 9 & 14 \\
Centro-Oeste & 10 & 15 \\
Sudeste & 32 & 48 \\
Sul & 13 & 20 \\
\hline Total & $\mathbf{6 6}$ & $\mathbf{1 0 0}$ \\
\hline
\end{tabular}

A maioria dos grupos de pesquisa está localizada na região Sudeste, com 32 grupos que correspondem a $48 \%$ do total. A segunda região em número de grupos é a Sul, que possui 13 grupos que corresponde a $20 \%$ do total, seguida da região Centro-Oeste e Nordeste. Os dados apresentados acima refletem, tanto o início do crescimento da Pós-Graduação em Enfermagem quanto a concentração de um maior número de Programas de Pós-Graduação em Enfermagem nas regiões Sudeste e Sul do País respectivamente.

Paralelamente a este crescimento, desde a criação em 1972 dos programas de mestrado e em 1981 dos programas de doutorado, observou-se um aumento na ordem de 69\% dos Programas de Pós-Graduação em Enfermagem credenciados pela Coordenação de Aperfeiçoamento de Pessoal de Nível Superior (CAPES), passando de 16 no triênio 1998-2000 para 27 no triênio 2004-2006. No período de 2001 a 2006 foram formados 2102 mestres e 653 doutores em Enfermagem oriundos dos diferentes estados brasileiros, países da América-Latina e da África de língua portuguesa. Atualmente estão credenciados 32 programas de Pós-Graduação. ${ }^{5}$ Entre as 29 instituições identificadas, 15 possuem Programa de Pós-Graduação Stricto Senso reconhecidos pela CAPES, sendo todas de caráter público federal ou estadual.

Observa-se neste sentido, que os grupos de Enfermagem que têm o foco em tecnologia dentre suas linhas de pesquisa, criados entre 1982 e 2008 , apresentaram um ritmo crescente, conforme mostra a Tabela 2.

Tabela 2 - Ano de formação dos grupos de pesquisa em enfermagem que se relacionam diretamente com a tecnologia

\begin{tabular}{lrr}
\hline Ano de formação do grupo & $\mathbf{n}$ & $\mathbf{\%}$ \\
\hline $1982-1988$ & 1 & 2 \\
$1989-1993$ & 8 & 12 \\
$1994-1998$ & 9 & 14 \\
$1999-2003$ & 20 & 30 \\
$2004-2008$ & 28 & 42 \\
\hline Total & $\mathbf{6 6}$ & $\mathbf{1 0 0}$ \\
\hline
\end{tabular}

Após a criação do primeiro grupo, em 1982, houve um intervalo de sete anos até a criação do grupo seguinte, quando a partir de então novos grupos foram criados. Os últimos quatro anos foram o período com a maior criação de grupos, com 28 grupos correspondendo a $42 \%$. 
Um aspecto que também pode ter influenciado o aumento na criação dos grupos de pesquisa desde 1999, foi o fato de que a partir de 2002, tornou-se obrigatório a todos os bolsistas de pesquisa, de mestrado, de doutorado, de iniciação científica, além de orientadores credenciados e outros sujeitos que utilizam o $\mathrm{CNPq}$, cadastrarem o currículo na Plataforma Lattes. Assim, os dados sugerem que o crescimento do número de grupos em 2002 pode ter ocorrido em decorrência da exigência das instituições de ensino para que os pesquisadores aumentassem o número de grupos de pesquisa com cadastro na Plataforma Lattes. De maneira geral, observamos que as modificações no sistema informacional do $\mathrm{CNPq}$ aumentaram a importância da plataforma para a visibilidade da pesquisa tanto no Brasil, quanto em nível internacional, além de fortalecerem o vínculo das instituições ao $\mathrm{CNPq}$, o que ocasionou uma maior exigência sobre os pesquisadores para a formação e cadastramento de grupos.

Com relação à distribuição dos grupos de pesquisa por estado, observa-se que São Paulo concentra o maior número de grupos (18), estando a maioria localizada na Escola de Enfermagem da Universidade de São Paulo (12), como ilustra a Figura 1.

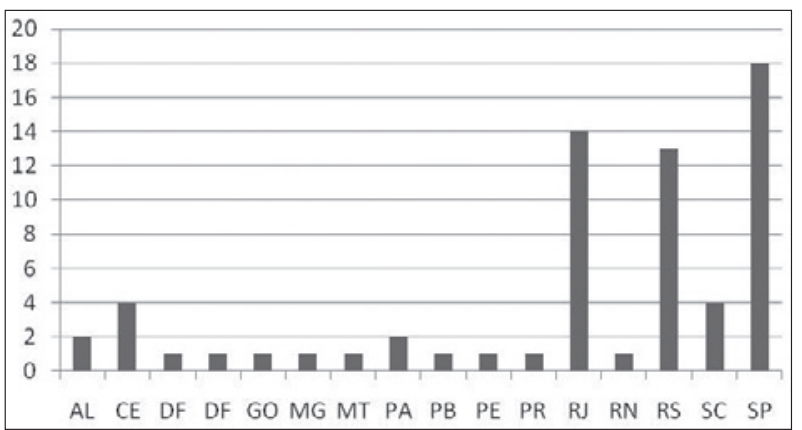

Figura 1 - Número de grupos de pesquisa em enfermagem com linha de pesquisa focada em tecnologia, por estado em 2008

O segundo estado em número de grupos é o Rio de Janeiro (14), sendo que estes se localizam na Universidade Federal do Rio de Janeiro, Universidade do Estado do Rio de Janeiro e Universidade Federal Fluminense. No Rio Grande do Sul, seis grupos estão na Fundação Universidade do Rio Grande, e os outros na Universidade de Caxias do Sul, Universidade Federal do Rio Grande do Sul, Universidade Luterana do Brasil, Universidade de Cruz Alta e Universidade de Passo Fundo. Santa Catarina e Ceará também se destacam em número de grupos, com quatro cada.
No que tange à distribuição do número de linhas por grupo de pesquisa em Enfermagem, relacionados à tecnologia, destaca-se que 52 grupos possuem de uma a três linhas de pesquisa, 12 possuem de quatro a cinco e dois possuem de sete a oito linhas de pesquisa.

Dentre os grupos identificados, sete têm entre suas linhas de pesquisa especificamente a Tecnologia da Informação e Comunicação (TIC). Neste estudo, as linhas de pesquisa são entendidas como temas aglutinadores de estudos científicos que se fundamentam em tradição investigativa, de onde se originam projetos cujos resultados guardam afinidades entre si. Portanto, a linha passa a ser a referência do grupo de pesquisa para a produção do conhecimento uma vez que está subordinada a ele. ${ }^{4}$

Ao se evidenciar a TIC como linha de pesquisa de destaque nos grupos de pesquisa identificados, cabe salientar que esta tem sido tema de pesquisa e reflexões em todo o mundo, especialmente por fatores como:

- a crescente evolução das TICs nas mais diversas formas e dispositivos que influenciam e mudam o mundo em que vivemos; ${ }^{6}$

- os desafios da saúde global para diminuir os erros e danos aos pacientes e, consequentemente, melhorar a segurança do paciente;

- a necessidade de diminuir a sobrecarga de trabalho dos profissionais de saúde;

- a necessidade de aproximar cada vez mais os profissionais de saúde para a evidência do cuidado também em áreas remotas e de difícil acesso com o foco voltado na Saúde para todos; 7,9

- a vasta amplitude de informação que desafia os profissionais para o desenvolvimento de um cuidado com qualidade e cada vez mais complexo; ${ }^{10}$

- a ampliação das inter-conexões e fronteiras profissionais que desafiam a progressão do trabalho interdisciplinar; 2,11

- a necessidade de valorizar e medir o capital intelectual da enfermagem; ${ }^{2}$

- a necessidade de se atentar para o conhecimento que os pacientes têm sobre a Enfermagem e a influência da tecnologia neste sentido. ${ }^{12}$

Desta forma, é possível chamar a atenção quanto à importância desta linha de pesquisa TIC em Enfermagem - e à sua produção como um caminho ao avanço do conhecimento em enfermagem e à melhoria da saúde da sociedade. 
Foram identificadas 59 linhas de pesquisa com denominações diferentes, embora ao sistematizá-las e categorizá-las, ${ }^{13}$ observou-se que $69 \%$ destas são assistenciais, $21 \%$ educacionais e $10 \%$ gerenciais.

Foram identificados 358 pesquisadores dentre os grupos consultados. Ao considerar o número de pesquisadores que integram os grupos, notou-se que 39 destes têm de um a quatro pesquisadores, 13 têm entre cinco e oito, 10 são integrados por nove a 12 pesquisadores e outros três grupos possuem 13 a 16 pesquisadores. Apenas um grupo apresentou acima de 17 pesquisadores.

Estes dados apontam a diversidade no número de pesquisadores nos diferentes grupos. Destaca-se neste sentido, os extremos entre o Núcleo de Estudos em Educação, Promoção em Saúde e Projetos Inclusivos (NESPROM), vinculado à Universidade de Brasília com 26 pesquisadores e outros nove diferentes grupos com apenas um pesquisador. Foi observado que a maioria dos grupos com um ou dois pesquisadores foram criados nos dois últimos anos e, grande parte deles, localizados na região Nordeste.

Ainda em relação aos componentes dos grupos de pesquisa, foi identificada a participação de 378 estudantes, em sua maioria de graduação (51\%), o que reforça a importância dessa, principalmente de iniciação científica. Dentre os demais estudantes, $33 \%$ eram alunos do curso de mestrado, $7 \%$ do curso de doutorado e $9 \%$ de especialização. Também foi identificada a presença de pessoal técnico, que contou com a participação de 170 profissionais, das mais diferentes áreas, embora o enfermeiro tenha sido o profissional predominante, o que também reforça a importância desta participação na melhoria da integração entre a pesquisa e a prática.

Além disso, destaca-se que em alguns grupos foi verificada a participação de pesquisadores de outras áreas do conhecimento e de outras instituições, em função possivelmente da complexidade do tema tecnologia, de sua característica multidisciplinar e da necessidade de ampliar o universo de aplicação e desenvolvimento dos produtos e resultados dos estudos nesta área.

O estudo também permitiu identificar que dentre os pesquisadores da enfermagem cadastrados nos grupos de pesquisa, $64 \%$ são doutores, $25 \%$ são mestres, e somente $11 \%$ são especialistas ou graduados. Em média são 3,46 doutores por grupo. Embora não se tenha uma análise concreta deste percentual de doutores por grupo em relação à produção do conhecimento, é importante destacar a participação dos doutores no desenvolvimento da pesquisa em enfermagem e também observar tal constatação como um reflexo do investimento dos Programas de Pós-Graduação em Enfermagem. Apenas sete grupos possuem algum tipo de relação com o setor produtivo, que em sua maioria, foi explicitada como relação com outras universidades públicas.

\section{CONSIDERAÇÕES FINAIS}

Entende-se que este estudo contribuiu principalmente, para dar maior visibilidade à produção de pesquisas em torno do tema tecnologia na enfermagem; para o conhecimento da distribuição espacial dos grupos de pesquisa em enfermagem de acordo com as diferentes regiões do Brasil; para refletir sobre a produção e o interesse dos pesquisadores nesta área nos diferentes níveis de formação; e, sobretudo, para promover o direcionamento da pesquisa nesta área de acordo com as necessidades brasileiras e o contexto internacional.

Como destaque, percebeu-se um crescente aumento dos grupos de pesquisa nos últimos anos com interesse em torno desta temática, além de uma tendência a aumentar os grupos de pesquisa em todas as regiões do país, não apenas de Sudeste e Sul.

Não há dúvidas que a tecnologia evolui a cada dia a um ritmo sem precedentes alcançando áreas remotas e de difícil acesso em todo o mundo, entretanto, ainda é incapaz de produzir mudanças no desenvolvimento de países mais pobres que enfrentam grandes desafios em termos de condições de saúde, emprego e vida decentes. A Enfermagem inserida neste contexto, enfrenta iguais desafios, pois ao mesmo tempo em que vivencia as mudanças tem também sua parcela de responsabilidade para ampliar e construir o capital intelectual da profissão no desenvolvimento de sua prática.

A pesquisa e produção do conhecimento nesta área é uma prioridade mundial. Quando realizada de modo coletivo sob a forma de grupos, torna-se um caminho efetivo para: traduzir os resultados dos estudos à prática profissional; ampliar a sua produção; dar maior visibilidade à enfermagem; integrar diferentes níveis de formação em um objetivo comum de avanço do conhecimento; e ampliar e desenvolver a capacidade de produção científica multidisciplinar.

Novos estudos se fazem necessários a partir dos resultados aqui apresentados, especialmente, 
no sentido de medir o impacto da produção dos grupos de pesquisa que trabalham com o foco tecnologia na prática de enfermagem para a melhoria do cuidado das pessoas.

\section{REFERÊNCIAS}

1. Martins CR, Dal Sasso GTM. Tecnologia: definições e reflexões para a prática em saúde e enfermagem. Texto Contexto Enferm. 2008 Jan-Mar; 17(1):11-2.

2. Simpson RL. Information technology building nursing intellectual capital for the information age. Nurs Adm Q. 2007 Jan-Mar; 31(1):84-8.

3. Erdmann AL, Lanzoni GMM. Caracteristicas dos grupos de pesquisa da enfermagem brasileira certificados pelo CNPq de 2005 a 2007. Esc Anna Nery Rev Enferm. 2008 jun; 12(2):316-22.

4. Marziale MH, Mendes IAC. A inserção social da Pós-Graduação em Enfermagem. Rev Latino-am Enfermagem. 2007 Set-Out; 15(5):883-4.

5. Conselho Nacional de Desenvolvimento Científico e Tecnológico (CNPq). Grupos de pesquisa: saiba mais. [acesso em 10 Dez 2008]. Disponível em: http://dgp.cnpq.br/diretorioc/html/faq.html

6. Abbott PA, Coenen A. Globalization and advances in information and communication technologies:
The impact on nursing and health. Nurs Outlook. 2008 Sep-Oct; 56(5):238-46.

7. Courtney KL, Demiris G, Alexander GL. Information technology changing nursing processes at the pointof-care. Nurs Adm Q. 2005 Oct-Dec:29(4):315-22

8. Zuzelo PR, Gettis C, Hansel AW, Thomas L. Describing the influence of technologies on registered nurses' Work. Clin Nurse Spec. 2008 May-Jun; 22(3):132-40.

9. Royal College of Nursing. E-Health: Putting the information at the heart of nursing care. How IT is set to revolutionise health care and the NHS. London (UK): NCH; 2006. p.1-8.

10. Bates DW, Gawande AA. Improving safety with information technology. N Engl J Med. 2003 Jun; 348(25):2526-34.

11. Simpson RL. No-borders nursing how technology heals global ills. Nurs Adm Q. 2004 Dec; 28(1):55-9.

12. Macdonald M. Technology and its effect on knowing the patient a clinical issue analysis. Clin Nurse Spec. 2008 May-Jun; 22(3):149-55.

13. Nietsche EA, Backes VMS, Colomé CLM, Ceratti $\mathrm{RN}$, Ferraz F. Tecnologias educacionais, assistenciais e gerenciais: uma reflexão a partir da concepção dos docentes de enfermagem. Rev Latino-am Enfermagem. 2005 Mai-Jun; 13(3):344-53. 